\title{
EL SISTEMA URBANO Y LAS ESTRATEGIAS DE LAS CIUDADES ASTURIANAS EN EL ESCENARIO DEL ARCO ATLANTICO
}

\author{
Lorenzo LÓPEZ TRIGAL
}

Desde 1989 en que fue lanzada la idea del Arco Atlántico por parte de los franceses, con la toma de conciencia de un espacio problemático y periférico en Europa como reacción de solidaridad de las regiones ("finisterres") y ciudades atlánticas frente a la "banana bleue" de Manchester a Milán, se han puesto en marcha diferentes equipos de estudios en el marco de la institución estatal francesa DATAR. Así en lo que concierne a su sección de Prospectiva y Territorios fue encargado en 1990 al Centre d'Études des Espaces Urbains (CESURB) de la Universidad de Burdeos III el análisis de la "Dinámica de las redes urbanas de la Fachada Atlántica", o el escenario de las nuevas centralidades de las ciudades atlánticas, dentro de un proyecto amplio acerca del futuro de esta macrorregión, liderado por el sociólogo Jacques Beauchard de la Universidad de Paris XII. Los trabajos de este grupo de investigadores son ya conocidos en tres publicaciones (BEAUCHARD, 1993, 1994,Y CHARRIÉ, LABORDE, 1993).

En esta misma línea, se ha prolongado el proyecto a las regiones españolas y portuguesas a lo largo del curso 1993-1994, con la participación de un grupo de profesores de Economía y de Geografía de España y Portugal, que éramos invitados por los colegas franceses a participar en el estudio de prospectiva sobre la Fachada Atlántica. Los resultados de las aportaciones serán publicados en 1995 en un volumen dirigido por el profesor J. BEAUCHARD y titulado "L'Espace-Projet Atlantique".

Es en este marco de reflexión y de debate acerca de la macrorregión de la fachada atlántica que se resalta en el enfoque del presente trabajo, en el que vamos a revisar, de un lado, la posición del sistema de ciudades de la región asturiana, que cuenta con una amplia aportación bibliográfica del grupo de geógrafos de la Universidad de Oviedo, iniciada ya en su tesis doctoral por el profesor EMILIO MURCIA $(1978,1980,1981)$, proseguida por otros como FERNÁNDEZ CUESTA Y FERNÁNDEZ PRIETO (1982, 1991); y, de otro lado, las dinámicas y estrategias que se plantean desde sus instituciones en relación al futuro de las ciudades y sus territorios, partiendo de la débil trama de interrelaciones entre las ciudades y regiones de la fachada atlántica ibérica y las rupturas que se advierten en su dilatado perímetro.

En la parte c sntral de la región de Asturias es donde está concentrada la mayor parte de su población (de 1.120.000 habitantes en el censo de 1991), diferenciándose en su territorio tres subregiones (de Occidente, Centro y Oriente), bien contrastadas por sus caracteres socioeconómicos además de geográficos. Mientras que las áreas occidental y oriental son más bien de carácter rural y periférico, es en la Asturias Central donde se han concentrado las poblaciones y los recursos de la región en la medida de la puesta a punto de las cuencas mineras del carbón, valles del Caudal y del Nalón sobre todo, los primeros núcleos de industrialización desde la implantación a fines del XVIII de Reales Fábricas de Armas en Trubia y en Oviedo, y desde mediados del XIX con la fundición de hierro en horno alto al coque en Trubia y en Mieres. Tras estas industrias pioneras, se desarrollará la industrialización de este siglo, por iniciativa tanto privada como pública, en torno a las ciudades, los puertos y las cuencas mineras, además de la densificación de las comunicaciones y la concentración de las actividades terciarias.

De este modo, las actividades minero industriales, primero, y las terciarias, después, han originado una organización del sistema territorial y urbano de Asturias muy peculiar, por su

Polígonos, n. ${ }^{\circ} 4$, 1994, pp. 107-114. 
concentración en el espacio central, con una trama de múltiples centros urbanos (las cinco ciudades de Gijón, Oviedo, Avilés, Mieres y Langreo (ésta en realidad es suma de asentamientos vecinos con un continuo urbano en la actualidad), además de villas y núcleos y asentamientos periurbanos de distinta talla, que viene a sumar unos 850.000 habitantes en la veintena de municipios que ocupan el rectángulo limitado por Castrillón (NO), Gijón (NE), Lena (SO) y Laviana (SE); ésto es, en un pequeño espacio se concentran tres cuartas partes de la población regional. Mientras que las dos zonas periféricas uccidental y oriental presentan una red de núcleos semiurbanos o villas y pueblos centro, que en ningún caso alcanzan la conocida talla urbana española de núcleos con más de diez mil habitantes.

Es preciso indicar en este punto el grado de diseminación del poblamiento de buena parte de los 78 municipios asturianos, donde a menudo la población del mayor núcleo, salvo en las tres mayores ciudades y en Langreo, no supera el 50 por ciento de la población municipal, al estar repartida la población lo más a menudo en numerosas entidades por cada concejo: Así, Mieres (48,8 \% la población de la localidad central y 491 entidades), Siero (18,9 \% y 193), o San Martin del Rey Aurelio, Castrillón, LLanes, Corvera de Asturias, Luarca, Aller, Cangas de Narcea, Villaviciosa y Laviana, por citar los municipios de más de quince mil habitantes. Relacionado con este fenómeno, hay a veces más de un lugar central en un mismo municipio; en Siero, por ejemplo, compiten Pola de Siero y Lugones; o bien se reduce la talla del núcleo central considerablemente a nivel de villa y de pueblo centro.

Por otra parte, el territorio del Área Metropolitana se extiende en, al menos, dieciseis municipios bajo la influencia directa de las cinco ciudades de mayor talla, cada una de ellas formando su propia área urbana. En este marco, corresponden cinco municipios a la comarca o subárea de Avilés (junto al municipio avilesino, los de Castrillón, Corvera, Gozón e Illas), dos a la subárea de Gijón (Carreño y Gijón), otros cinco a la subárea de Oviedo (LLanera, Ribera de Arriba, Noreña, Oviedo y Siero), tres a la subárea de Langreo (Laviana, Langreo, San Martin del Rey Aurelio), y el único municipio de la subárea de Mieres, si bien conectan con ella los municipios de Lena (Pola de Lena) y Aller (Cabañaquinta y Moreda).

En la anterior jerarquía de los lugares centrales de Asturias, ordenada por unidades de cuota de mercado sobre la base 100.000 del conjunto de España, puede constatarse la presencia de cuatro niveles de centralidad, correspondientes a la talla de ciudades medias grandes, ciudades medias pequeñas, núcleos periurbanos y pequeñas ciudades, y villas. El denominado sistema urbano asturiano es en realidad un subsistema del propio sistema urbano español, liderado a nivel VII de la jerarquía por la gran ciudad de Madrid, de la que dependen los centros asturianos.

En primer lugar, en el nivel $\mathrm{V}$ superior de la región, destacan como ciudades intermedias grandes o ciudades de alcance regional, Gijón y Oviedo, con una semejante cuota de mercado, 590 y 524, aunque con ciertas diferencias de talla poblacional, y correspondiendo en la jerarquía de las ciudades españolas según esta misma cuota a los puestos 18 y 19 respectivamente, por detrás de otras ciudades de la fachada atlántica española como Bilbao, el mayor centro de la misma, (en un nivel VI y cuota de 955 unidades), o las capitales gallegas del nivel V, Vigo (cuota de 742) y La Coruña (cuota de 705), y por delante, en cambio, de otras del mismo nivel V como Santander (cuota de 501), San Sebastián (493), Vitoria (489) o Pamplona (488).

Como ciudades medias pequeñas, nivel IV, se presentan en el sistema urbano asturiano, las de Avilés, Mieres y Langreo. Sobresale la ciudad de Avilés en este nivel, con 192 de cuota mercado y una talla poblacional próxima a los cien mil habitantes, mientras a cierta distancia se presentan las otras dos ciudades, a su vez muy emparejadas por los datos que se observan, por su respectivas cuotas de mercado de 117 y 109 y talla poblacional municipal, como por su especialización funcional en la minería.

El nivel básico urbano, o nivel III de las pequeñas ciudades, podríamos convenir que está ausente en el sistema de Asturias, pues aunque contemos con localidades de cuatro municipios del Área Metropolitana insertos en este nivel, más bien se trata de núcleos periurbanos y barriadas ubicadas en municipios vecinos a las ciudades de las que se hallan estrechamente dependientes. Es el caso de las localidades de Lugones y Pola de Siero respecto de Oviedo, de El Entrego y Sotrondio en relación a la ciudad de Langreo, y en dos municipios de la comarca de Avilés, los núcleos barriadas de Piedras Blancas y Salinas al noroeste, y de Las 
Vegas al suroeste de Avilés. En todos ellos, salvo en el caso de Pola de Siero, son núcleos dormitorio que se han desarrollado en paralelo al crecimiento de la ciudad matriz y como contínuo urbano de cada una de ellas.

\section{JERARQUÍA URBANA Y DE LUGARES CENTRALES}

\begin{tabular}{|c|c|c|c|c|c|}
\hline nivel & rango & municipio - localidades & cuota mercado & $\begin{array}{r}\% \text { población mayor } \\
\text { núcleo }\end{array}$ & población total \\
\hline \multirow[t]{2}{*}{$\mathrm{V}$} & 1 & Gijón & 590 & 94,6 & 260,2 \\
\hline & 2 & Oviedo & 524 & 87,8 & 203,1 \\
\hline \multirow[t]{3}{*}{ IV } & 3 & Aviles & 192 & 92,9 & 84,0 \\
\hline & 4 & Mieres del Camino & 117 & 48,8 & 53,0 \\
\hline & 5 & Langreo & 109 & 80,2 & 51,3 \\
\hline \multirow[t]{4}{*}{ III } & 6 & $\begin{array}{l}\text { Siere (2)-Lugones y } \\
\text { Pola de Siero }\end{array}$ & 110 & 18,9 & 45,2 \\
\hline & 7 & $\begin{array}{l}\text { San Martin Rey Aure- } \\
\text { lio (5)- E1 Entrego y } \\
\text { Sotrondio }\end{array}$ & 47 & 31,3 & 31,3 \\
\hline & 8 & $\begin{array}{l}\text { Castrillón (3)- Piedras } \\
\text { Balncas y Salinas }\end{array}$ & 44 & 33,9 & 21,2 \\
\hline & 9 & $\begin{array}{l}\text { Carvera de Asturias } \\
\text { (3)-Vilalegre }\end{array}$ & 40 & 48,4 & 17,0 \\
\hline \multirow[t]{8}{*}{ ILa } & 10 & LLanes & 41 & 27,4 & 13,3 \\
\hline & 11 & Luarca & 38 & 25,3 & 16,6 \\
\hline & 12 & Cangas de Narcea & 36 & 36,1 & 19,0 \\
\hline & 13 & Aller-Moreda & 36 & 18,0 & 17,3 \\
\hline & 14 & Lema - Pola de Lena & 33 & 57,2 & 13,8 \\
\hline & 15 & Villavicioen & 33 & 29,3 & 15,0 \\
\hline & 16 & $\begin{array}{l}\text { Laviana (5)- Pola de } \\
\text { Laviana }\end{array}$ & 32 & 44,0 & 15,1 \\
\hline & 17 & Grado & 30 & 47,2 & 12,0 \\
\hline \multirow[t]{16}{*}{ IIb } & 18 & Tineo & 29 & 18,4 & 14,8 \\
\hline & 19 & Navia & 28 & 32,5 & 8,8 \\
\hline & 20 & Gozón (3)- Luanco & 24 & 42,3 & 11,4 \\
\hline & 21 & LLanera (2)- Posada & 24 & 19,3 & 10,4 \\
\hline & 22 & Pravia & 24 & 41,8 & 9,9 \\
\hline & 23 & Piloria - Infiesto & 22 & 20,0 & 9,5 \\
\hline & 24 & Carreño (1) - Candás & 21 & 51,2 & 11,0 \\
\hline & 25 & Salas & 20 & 18,0 & 8,0 \\
\hline & 26 & Cangas de Onis & 18 & 45,1 & 6,4 \\
\hline & 27 & Cudillero & 18 & 31,3 & 6,5 \\
\hline & 28 & Ribadesella & 17 & 53,6 & 6,1 \\
\hline & 29 & Colunga & 15 & 24,3 & 4,9 \\
\hline & 30 & Vegadeo & 15 & 53,5 & 5,0 \\
\hline & 31 & Noreĩa (2) & 14 & 97,6 & 4,1 \\
\hline & 32 & Parres - Arriondas & 13 & 40,1 & 5,7 \\
\hline & 33 & Nava & 13 & 30,1 & 5,7 \\
\hline
\end{tabular}

Fuente: Elaboración propia a partir del Anuario del Mercado Español. 1992. Madnd, Banesto, 1992, pp. 272-275.

Nota: Se recoge la población de hecho del Censo de 1991, en miles de habitantes y la cuota de mercado, calculada por el Anuario de Banesto que resume la capacidad mercadológica de cada municipio. Se anotan, por otro lado, con un número entre paréntesis, aquellos municipios que quedan bajo la influencia directa de un núcleo de mayor rango jerárquico en el Área Metropolitana, así Siero con relación a Oviedo va con el número del rango 2 correspondiente.

Frente a la ausencia de hecho de las pequeñas ciudades, es generalizable la presencia de una larga veintena de villas distribuídas por las subregiones central, occidental y oriental del espacio asturiano. Hasta el punto que esta diversidad entendemos que tiene una cierta distinción interna en su nivel correspondiente, presentando en la jerarquía de lugares centrales no urbanos, un primer subgrupo II a, c.Jmpuesto de seis villas, tradicionales cabeceras de comarca, como LLanes, Luarca, Cangas de Narcea, Pola de Lena, Villaviciosa y Grado, más otras dos poblaciones de crecimiento más reciente ubicadas en la cuenca minera central, Pola de 
Laviana, que aunque población con mercado tradicional como las anteriores villas se suma a la localidad de Moreda como núcleos de hábitat esencialmente minero. Y, por otro lado, un segundo subgrupo II b, formado tanto por pequeñas poblaciones periurbanas del Área Metropolitana, como Posada y Noreña en relación a Oviedo, cuanto pequeñas cabeceras, enclavadas en el litoral, villas costeras de Vegadeo, Navia, Cudillero, Luanco, Candás, Colunga y Ribadesella, o en el interior occidental, Tineo, Pravia y Salas, e interior oriental, Infiesto, Cangas de Onís, Arriondas y Nava.

Al igual que en las otras regiones de la fachada atlántica, en Asturias está generalmente bien trabada esta malla de villas. Villas costeras, en unos casos, donde la función del pequeño puerto y la pesca se complementan con las funciones terciarias, el turismo veraniego, y aún con la función residencial las ubicadas en el Área Metropolitana o su vecindad (Candás, Luanco, Cudillero). En el caso de las de mayor centralidad como Luarca y LLanes destacan como grandes villas con una centralidad conservada por su distancia respecto de las ciudades del área central. En otros casos, se trata de villas del territorio interior asturiano, que organizan las comarcas y que han evolucionado de ser anteriormente puntos de encrucijada y de mercados a ser actualmente centros terciarios más o menos especializados, como es el caso de Cangas de Narcea en la actividad minero-energética y Cangas de Onís, en el turismo del parque nacional de Picos de Europa.

En síntesis, el sistema urbano de Asturias se caracteriza básicamente, en primer término, por el dominio del Área Metropolitana, enclavada en el espacio central con la presencia de cinco ciudades, por tanto área de tipo policentral, y de otra decena de poblaciones vecinas de reciente crecimiento periurbano y de villas tradicionales renovadas por la demanda de nuevos usos y espacios, y, en segundo término, por la ausencia del nivel intermedio de la pequeña ciudad, sobre todo en los espacios periféricos ya que en el Área Metropolitana se ve suplida la ausencia por núcleos periurbanos.

La prospectiva del sistema urbano así caracterizado sigue las trazas del pasado reciente en cuanto a la concentración de población y funciones urbanas e industriales en torno al espacio del Área Metropolitana, donde se asientan las mayores ciudades y prácticamente la totalidad de los espacios industriales, tal como se refleja en la gráfica. En estos espacios se aprecia la gran extensión ocupada por las dos factorías de la empresa siderúrgica pública ENSIDESA, con un espacio industrial localizado al Este de Avilés, en los años 50 y posteriores, y al Oeste de Gijón, a fines de los años 60; junto a la difusión reciente de polígonos industriales para la pequeña y mediana industria, además de localizaciones puntuales notables como la última inversión de la multinacional DU PONT al sur de Avilés, cierta empresa pública del INI en Avilés o Trubia (municipio de Oviedo); las explotaciones mineras de carbón de HUNOSA en los valles de Mieres y de Langreo; una industria en declive como la DURO FELGUERA en Langreo; o algunas instalaciones de centrales térmicas en Mieres, Langreo, Aboño (GijónCarreño) y Soto de Ribera (al sur de Oviedo). En esta situación, el espacio del Área Metropolitana está incrementando su cohesión a través de las mejoras de infraestructuras de transportes y comunicaciones, con flujos intensos entre las distintas localidades, siendo de hecho la capital política regional, Oviedo, la que mejor renta de situación mantiene en el Área.

Después de la anterior revisión del sistema urbano, analicemos otros aspectos esenciales en la organización espacial de esta región, tales como la situación demográfica y la situación económica y social. En primer lugar, la dinámica actual de la población asturiana es de tipo estacionario, en cuanto a la evolución de su crecimiento natural como real, situada en la etapa finitransicional, al igual que sucede en la población española, ésto es, un estancamiento del índice de crecimiento situado a un nivel próximo a cero, donde los saldos migratorios coinciden también en su carácter equilibrado. En todo caso, mientras sigue la tónica de despoblación de los núcleos rurales de la subregiones periféricas oriental y ocidental así como todo el borde sur montañoso, se suma ahora también a la tendencia de despoblación las cuencas mineras, cuya población al igual que la emigrante de las zonas rurales va a confluir mayoritariamente en las ciudades de Oviedo y Gijón (sobre todo de personas jubiladas) y en especial en los núcleos periurbanos de Oviedo y de Avilés (en especial de personas jóvenes), caso de Castrillón, que ha sido el municipio con un mayor índice de crecimiento demográfico durante el último periodo intercensal y donde las notables expectativas demográficas animan a ampliar recientemente en el Plan General de Ordenación Urbana el suelo urbano de los 
núcleos de Piedras Blancas y de Salinas.

En segundo lugar, la situación económica y social asturiana está caracterizada por la grave crisis que atraviesan las empresas del Instituto Nacional de Industrial, de un peso muy importante en la estructura industrial de la región y que supone la de una mayor participación del INI entre las regiones españolas. Esa crisis actual ha supuesto la disminución del personal empleado de forma drástica con las jubilaciones anticipadas y despidos hasta alcanzar la cifra de doce mil empleos menos solamente en las previsiones de las dos mayores empresas ENSIDESA Y HUNOSA. Las perspectivas a corto y medio plazo presentan, de un lado, la misma tendencia de declive de la industria pública, con la previsión de la desaparición de empresas como Santa Bárbara en Oviedo y de industrias subsidiarias para estas industrias básicas, pero también, del lado del sector primario, se suma la crisis de las explotaciones agrario ganaderas y pesqueras como en el resto de la fachada atlántica española. Por todo lo cual, la región presenta altos índices de deterioro de la economía y del empleo, entrando en crisis el concepto de "Asturias como taller del Estado" y de "región industrial en declive" y afianzándose lo que para Manuel Castells (EL PAís, 16 agosto 1994) se denominaría el "síndrome de Asturias" al igual que otras regiones europeas, como paradigma de regiones desarticuladas e inadaptadas a los nuevos ritmos del mercado.

La anterior situación, evidentemente, afecta más seriamente a la evolución reciente de las ciudades y villas mineras e industriales asturianas, de ahí la preocupación e interés de las instituciones y asociaciones locales como de la región en esta materia. Así, en primer término, por parte del Principado de Asturias se ha presentado en 1994 un Programa de Industrialización y Dinamización Económica (PREDEA), basado en estudios recientes, que pretende dirigir, coordinado con el resto de las Administraciones, las líneas del crecimiento de la región hacia nuevas implantaciones industriales, la terciarización y la atracción del turismo, así como a la formación y a nuevas infraestructuras. Se aspira con ello al reto de salir del síndrome y alcanzar el umbral de la competitividad en condiciones similares a otras regiones, de incorporarse a la vez al proyecto de red de regiones y ciudades del Arco Atlántico.

En mayor o menor grado se ha diseñado también para cada una de las ciudades del Área Metropolitana un Plan Estratégico, con objetivo en el año 2000 o la próxima década, a falta de una estrategia de desarrollo para todo el conjunto del Área. Los retos actuales de ésta se encuentran en relación, primero, con el proceso de reestructuración económica de la región, y, segundo, el proceso de integración en las redes de las ciudades europeas, según las propias manifestaciones de uno de los principales agentes, el alcalde de Gijón, Álvarez de Areces, quien añade que es simplista pensar que la industria corresponde a una ciudad (Avilés o Gijón) y los servicios a otra (Oviedo).

Para el municipio de Oviedo, la estrategia local, como para el resto de ciudades, ha sido durante estos años impulsar el equipamiento público de la ciudad, y en este caso además la mejora de la imagen urbana, rivalizando con otras ciudades como Gijón, a nivel del patrimonio cultural y artístico de su casco antiguo y ensanche, impulso de la Universidad, del área de investigación y del parque tecnológico, entre otras actuaciones.

En Gijón, ciudad con problemas industriales por la reconversión siderúrgica y naval y de accesibilidad en comunicaciones por ferrocarril y carretera a lo largo del litoral, el diseño y reordenación de su espacio económico y urbano tiene por objetivo contribuir a la recuperación industrial y del empleo, la promoción de una dimensión cultural y universitaria (compitiendo directamente con Oviedo) y de imagen urbana a través de la mejora del medio ambiente y del potencial de los sectores comercial y turístico (donde confluyen ejes a promocionar como la ruta de la plata (Norte-Sur) y la costa verde (Este-Oeste).

En el caso de Avilés, junto a los municipios de su comarca, apunta a un escenario donde se destaca la potenciación de su vecindad al aeropuerto regional, el mismo puerto industrial pesquero y también desde ahora deportivo, la disposición de un amplio espacio industrial tras la liberación de parte de los terrenos de ENSIDESA, la mejora del medio ambiente, y el fomento del turismo litoral (imagen que detenta Salinas) y de la villa antigua avilesina.

Para las ciudades y villas mineras se plantea la mejora de la calidad de vida especialmente a partir del realojamiento en nuevas residencias, de nuevos equipamientos públicos y de un terciario con muchas deficiencias hasta ahora, además de la reordenación y aprovechamiento del suelo industrial. Destaca en este sentido, la operación en proceso del llamado "nuevo Lan- 
greo", que consta de un proyecto de ordenación del territorio en este sector del valle del Nalón, en materia urbanística, de infraestructuras y comunicaciones, junto a actuaciones ambientales.

De este modo se puede sintetizar actualmente la respuesta institucional regional y local a la crisis industrial y urbana asturiana, que, sumada a las directrices de la Administración Central y a la propia ordenación territorial y urbana de la región y de los municipios, nos presenta un interés decidido en pro de nuevas líneas de actuación y de estrategias para dar salida a los problemas actuales. Resta si cabe la respuesta de la sociedad y en especial de la empresa privada, tan ansiada si es extranjera como se ha comprobado en un asunto crítico que afectó a la misma Presidencia de la Comunidad Autónoma en 1993, pues, como ya advertía hace dos siglos el político Jovellanos, Asturias "carece de capitalistas". Pero tampoco cabe esperar sin más la resolución positiva de los problemas acumulados, sin apoyo de la Administración y en manos de la tendencia del mercado, ya que eso abocaría a una nueva etapa de emigrantes asturianos.

Como reflexión última añadiría, en primer término, que la articulación del subsistema urbano de Asturias con el de Cantabria y País Vasco al Este y el de Galicia al Oeste es esencial para la conectividad y trabazón de la fachada atlántica española e ibérica a su vez con la francesa, sin esa soldadura permanecerían las rupturas que se advierten todavía en los flujos entre ciudades y regiones de la fachada. Mientras tanto, Asturias sigue necesitando de una mayor accesibilidad al subsistema urbano de la España interior, en especial de Castilla y León, que sirve en buena medida de espacio de unión de Asturias por su borde sur con las ciudades y regiones europeas. En otro orden, la crisis paradigmática del caso de la economía asturiana encierra todo un desafío para los próximos años, tanto para la región como en espe cial para las ciudades y en particular el Área Metropolitana asturiana, en la búsqueda de una nueva imagen urbana e industrial. 


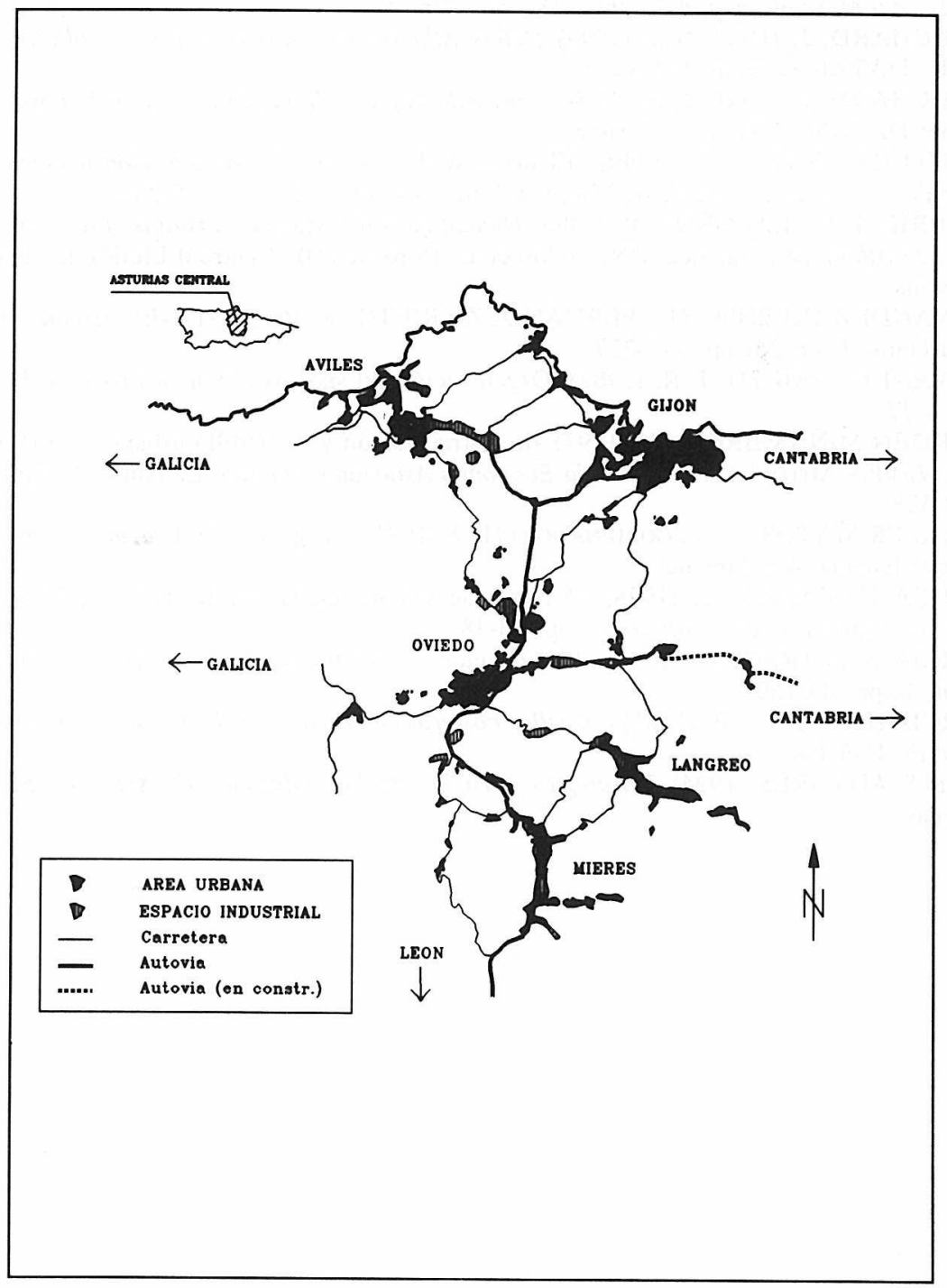




\section{BIBLIOGRAFÍA}

Anuario del Mercado Español. 1992. Madrid: Banesto.

BEAUCHARD, J. (DIRECTOR) (1993) Detins Atlantiques: entremémoire et mobilité. Mar seille: DATAR-Éditions de l'Aube.

BEAUCHARD, J. (DIRECTOR) (1994) Ciés Atlantiques: l'invention de la ville-pays. Mar seille: DATAR- Éditions de l'Aube.

BENITO DEL POZO , P. (1990) «El declinar de los espacios minero-siderúrgicos tradi cionales. Evolución reciente de Mieres y Langreo». Ería, 23, pp. 235-252.

CHARRIÉ, J.-P., LABORDE, P. (1993) Dynamiques des systèmes urbains et devenir de la Façade Atlantique. Talence: CNRS-Université Bordeaux III, Centre d'Études des Espaces Urbains.

FERNÁNDEZ CUESTA, G., FERNÁNDEZ PRIETO, J. R. (1991) «El sistema urbano asturiano». Ería, 26, pp. 217-227.

FERNÁNDEZ PRIETO, J. R. (1982) «Organización del sistema urbano asturiano». Ería, 3, pp. 5-77.

LLORDEN MIÑAMBRES, M. (1994) «Industrialización y desarrollo urbano en Asturias». En: VARIOS AUTORES Historia de la Economía Asturiana. Oviedo: La Nueva España, pp. 513-5 $\simeq 8$.

MORALES MATOS, G. (COORDINADOR) (1991-1993) Geografia de Asturias. Oviedo: La Nueva España, 4 volúmenes.

MURCIA NAVARRO, E. (1978) "Análisis de sistema en Geografía Urbana: El sistema urbano asturiano". El Basilisco, 5, pp. 33-38.

MURCIA NAVARRO, E. (1980) "Introducción al estudio del sistema urbano asturiano". Ería, 1, pp. 89-150.

MURCIA NAVARRO, E. (1981) Las villas costeras en el sistema urbano asturiano. Oviedo: Silverio Cañada.

VARIOS AUTORES (1994) Estrategias para la reindustrialización de Asturias. Madrid: Cívitas 